\title{
Self-assembling of $\mathrm{Ge}$ on finite $\mathrm{Si}(001)$ areas comparable with the island size
}

\author{
L. Vescan, ${ }^{\text {a) }}$ T. Stoica, and B. Holländer \\ Institut für Schichten und Grenzfächen (ISG), Forschungszentrum Jülich GmbH, D-52425 Jülich, Germany
}

\author{
A. Nassiopoulou, A. Olzierski, and I. Raptis \\ NCSR Demokritos Institute of Mikroelectronics, 15310 Aghia Paraskevi, Athens, Greece \\ E. Sutter \\ Department of Physics, Colorado School of Mines, Golden, Colorado 80401
}

(Received 18 February 2003; accepted 21 March 2003)

\begin{abstract}
Voids in ultrathin oxide and electron-beam lithography-patterned windows were used to deposit Ge selectively. The number of islands is a function of the total amount of $\mathrm{Ge}$ deposited in a void or window. Our results show that islands smaller than the void/window size nucleate mainly near the periphery. This might be due to the tensile strain in the $\mathrm{Si}$ substrate near the oxide edge. The interruption of the wetting layer reduces the loss of excitons by lateral diffusion, resulting in considerable increase in optical emission from islands. (c) 2003 American Institute of Physics.
\end{abstract}

[DOI: $10.1063 / 1.1576498]$

The use of Stranski-Krastanow (SK) growth mode in integrated circuits requires an accurate control over the spatial positioning of islands. The control of single $\mathrm{Ge}$ island positioning using selective epitaxial growth (SEG) was recently demonstrated for small windows formed in oxide by electron-beam lithography. ${ }^{1}$ On the other hand, $\mathrm{Si}$ and $\mathrm{Ge}$ grow selectively even if the oxide is as thin as $0.3 \mathrm{~nm} .^{2}$ Growth was performed in this case in voids formed by the partial thermal decomposition of the oxide. The thermal decomposition of oxides is a phenomenon which depends on temperature, time, and oxide thickness as investigated by Liehr et al. ${ }^{3}$ For 5-50-nm-thick oxides with large voids (1$100 \mu \mathrm{m})$ they found that the growth of void size proceeds with an activation energy of $2 \mathrm{eV}$ and somewhat slower than inversely proportional to the oxide thickness. Moreover, after the nucleation phase of the oxide decomposition the void size increases approximately linearly with time while the void density remains roughly constant. ${ }^{3}$ Radio Corporation of America (RCA) cleaned Si wafers have a 1-2-nm-thick oxide. This ultrathin oxide (UTO) is usually removed before epitaxy by thermal decomposition (the so called in situ cleaning step at high temperature). Voids in UTO offer the advantage of an easy procedure to study the nucleation of $\mathrm{Ge}$ by selective epitaxy in a large range of void size simultaneously. In particular, the Ge wetting layer is automatically interrupted if the oxide is only partially removed.

In this letter we examine the role of size of deposition area on self-assembling of Ge by selective epitaxial growth on areas of the order of the island size. Voids in ultrathin oxides and electron beam lithography (ebeam) patterned wafers were used as substrates. We show that the nucleation and morphology of $\mathrm{Ge}$ islands depends on the area size and that excellent optical emission is obtained if the wetting layer is not continuous.

\footnotetext{
a) Author to whom correspondence should be addressed; electronic mail: 1.vescan@fz-juelich.de
}

Experiments were performed by SEG in a low pressure chemical vapor deposition system with a base pressure of 8 $\times 10^{-8}$ Torr. Unpatterned and thermally oxidized $\mathrm{Si}(001)$ wafers patterned with ebeam were used. Our ex situ cleaning is based on a dilute RCA chemistry ${ }^{4}$ which results in the formation of a chemical UTO. After loading the wafers into the episystem the unpatterned wafers were treated at $875^{\circ} \mathrm{C}$ for $10 \mathrm{~min}$ so that the oxide breaking was just started, while the UTO of the ebeam wafers was completely removed at $900{ }^{\circ} \mathrm{C}$ for $10 \mathrm{~min}$. Ge deposition was performed at $700^{\circ} \mathrm{C}$ with a growth rate of $\sim 2$ equiv.ML $\min ^{-1} .^{5}$ The samples were examined by atomic force microscopy (AFM) and photoluminescence spectroscopy (PL). The Ge coverage was determined by Rutherford backscattering.

Figure 1(a) shows an AFM image of Ge islands deposited by selective epitaxy of $0.58 \mathrm{~nm}$ Ge (see also Table I). Growth proceeds only in the voids opened in UTO during the high temperature step. Voids of various sizes ranging from 50 to $260 \mathrm{~nm}$ can be seen in this image. The shape of the voids is square with rounded corners and parallel to $\langle 110\rangle$ directions. In voids, self-assembling of Ge occurs if the coverage exceeds a certain value. Moreover, the number of islands depends on the void area. Only one island is formed if voids are smaller than $\sim 270 \mathrm{~nm}$. This is consistent with the findings of Kim et al. ${ }^{1}$ and Nguyen et al. ${ }^{6}$ The islands are pyramids, as clearly seen by their square shape oriented $45^{\circ}$ to the void edges. In ebeam patterned windows slightly higher coverage leads to the formation of pyramids, too [Fig. 1(b)]. The number of pyramids in this case is higher because the windows are larger $(520 \mathrm{~nm})$. Further increase of coverage to $1.26 \mathrm{~nm}$ does not increase the number of islands immediately, it leads first to an increase of the island volume and to a shape transition (sample No. 5 in Table I). Windows of $270 \mathrm{~nm}$ size with a coverage leading to dome formation reveal one big dome and sometimes a small island nearby [Fig. 1(c)].

An interesting observation, in agreement with literature ${ }^{1}$ 
(a)

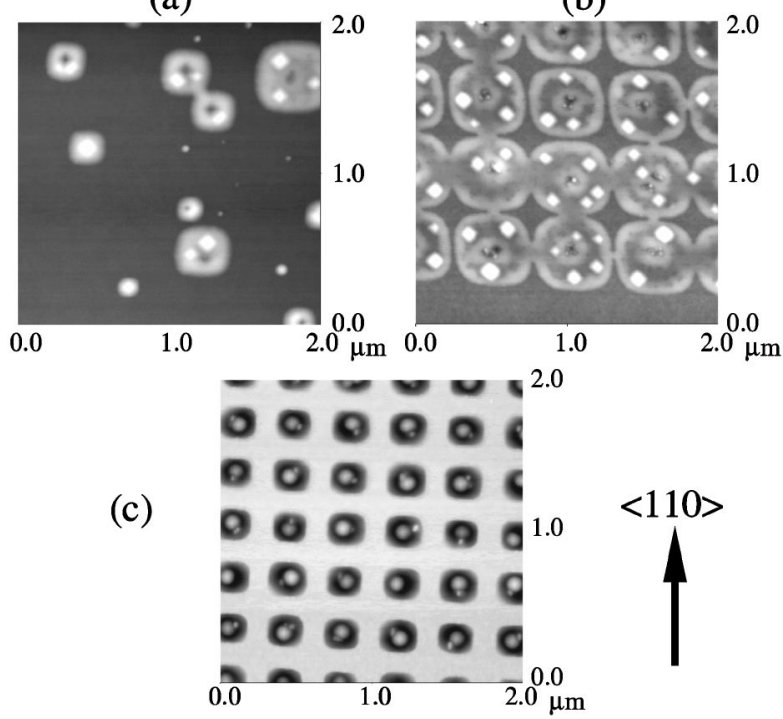

FIG. 1. AFM images of Ge islands: (a) deposition only in voids in ultrathin oxide (sample No. 1), (b) deposition in $520 \mathrm{~nm}$ large windows in ebeam patterned oxide (sample No. 4), and (c) $270 \mathrm{~nm}$ windows in ebeam patterned windows (sample No. 3).

is that, islands nucleate mainly near the periphery of the voids [Figs. 1(a) and 1(b)], similar to the ordered rows of islands on mesa edges. ${ }^{7,8}$ In general, a discontinuity of a surface film gives rise to an edge force. ${ }^{9}$ Nucleation of Ge at the periphery of the voids and windows would indicate that the Si substrate is tensile strained near the oxide edge. We explain this phenomenon by the higher expansion coefficient of $\mathrm{Si}$ as compared to $\mathrm{SiO}_{2}$ which makes $\mathrm{Si}$ to contract more than the oxide when cooled from high temperature to $700{ }^{\circ} \mathrm{C}$. Thus, Si will be tensile strained near the edge, and consequently, compressively strained in the center. Moreover, the compressive strain in the center of the void can explain the occurence of $\mathrm{SiC}$ dots in the center of voids when carbon contamination is present. ${ }^{10}$

In order to understand the evolution of island number with void size we calculated the Ge thickness for a certain void size which can be redistributed into one or more islands in the void. This model is based on the analysis of size distribution of islands described in Fig. 1 and Table I. Figure 2 displays the calculated curves and the experimental points of Ge thickness versus void size. For the calculated lines we have assumed that the two-dimensional coverage of thickness $d$ in a void or window of size $l$ leads (above the SK transition) to the formation of $N$ islands of volume $N^{*} V_{\text {isl }}$ $=l^{2 *}\left(d-d_{\mathrm{WL}}\right)$, where $d_{\mathrm{WL}}$ is the thickness of the wetting

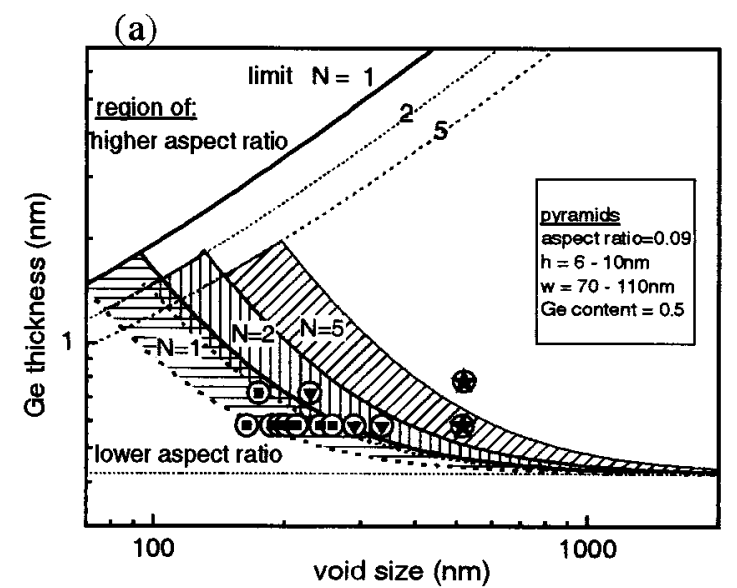

(b)

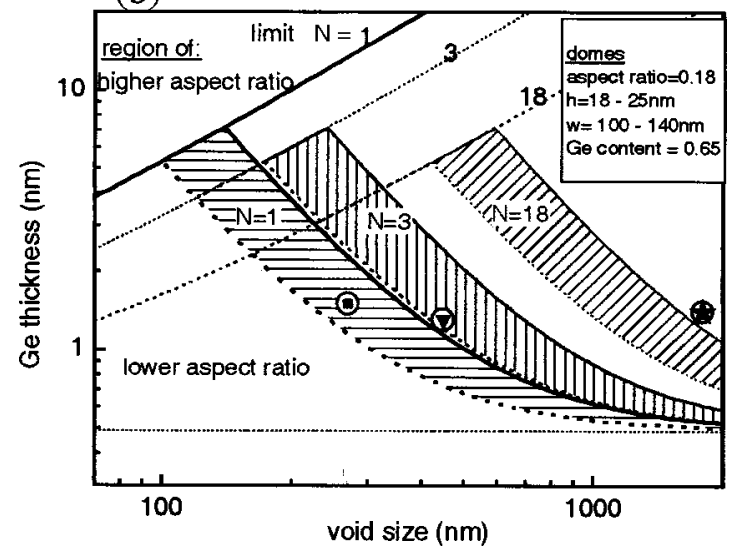

FIG. 2. Calculated equivalent Ge layer thickness necessary to produce one or several islands vs void or window size. (a) Pyramids with height $h$ $=6-10 \mathrm{~nm}$, width $w=70-110 \mathrm{~nm}$, and Ge content 0.50 , (b) domes with $h=18-25 \mathrm{~nm}, w=100-140 \mathrm{~nm}$, and Ge content of 0.65 . The points are experimental values; full squares: one island, triangles: two islands, and stars: five islands for (a) and eighteen islands for (b).

layer around the islands. For the volume of the pyramid we have taken the known formula. For domes we approximated the shape with a spherical calotte. Below SK transition $\left(h_{\mathrm{SK}}\right)$ the deposition leads usually only to the formation of a thin lattice matched wetting layer. This seems to be also the case for the void sizes studied here, as revealed by transmission electron microscopy analysis (not shown here). Figure 2(a) is for pyramids and Fig. 2(b) for domes. For each number of islands a range of island sizes was taken. For instance in Fig. 2(a) for $N=1$ the lower curve corresponds to island width $w=70 \mathrm{~nm}$ and height $h=6 \mathrm{~nm}$, while the upper curve is for $w=110 \mathrm{~nm}$ and $h=10 \mathrm{~nm}$. The limit lines correspond to the thickness at which the island fills the void (i.e., $w=l$ ). For

TABLE I. Data for Ge islands nucleated in voids and windows; $P$-pyramid, $D-$ dome, $N_{\text {isl }}-$ number of islands per window, and UTO—ultrathin oxide with voids.

\begin{tabular}{cccccccc}
\hline \hline $\begin{array}{c}\text { Epitaxy } \\
\text { No. }\end{array}$ & $\begin{array}{c}\text { Void } \\
\text { size } \\
(\mathrm{nm})\end{array}$ & Pattern & $\begin{array}{c}\mathrm{Si} \\
\text { buffer } \\
(\mathrm{nm})\end{array}$ & $\begin{array}{c}\mathrm{Ge} \\
(\mathrm{nm})\end{array}$ & $\begin{array}{c}\mathrm{Si} \\
\text { cap } \\
(\mathrm{nm})\end{array}$ & $\begin{array}{c}\text { Shape } \\
\text { of isl. }\end{array}$ & $N_{\text {isl }}$ \\
\hline 1 & $40-700$ & UTO & 5 & 0.58 & 0 & $P$ & $0-6$ \\
2 & $100-230$ & UTO & 0 & 0.72 & 0 & $P$ & $0-2$ \\
3 & 270 & ebeam & 0 & 1.45 & 0 & $D$ & $1-2$ \\
4 & 520 & ebeam & 5 & 0.78 & 0 & $P$ & $3-5$ \\
5 & 520 & ebeam & 5 & 1.26 & 0 & $D$ & $3-5$ \\
6 & 1800 & Opt.lith. & 5 & $\sim 1.4$ & 0 & $D$ & 18 \\
\hline \hline
\end{tabular}


(a)
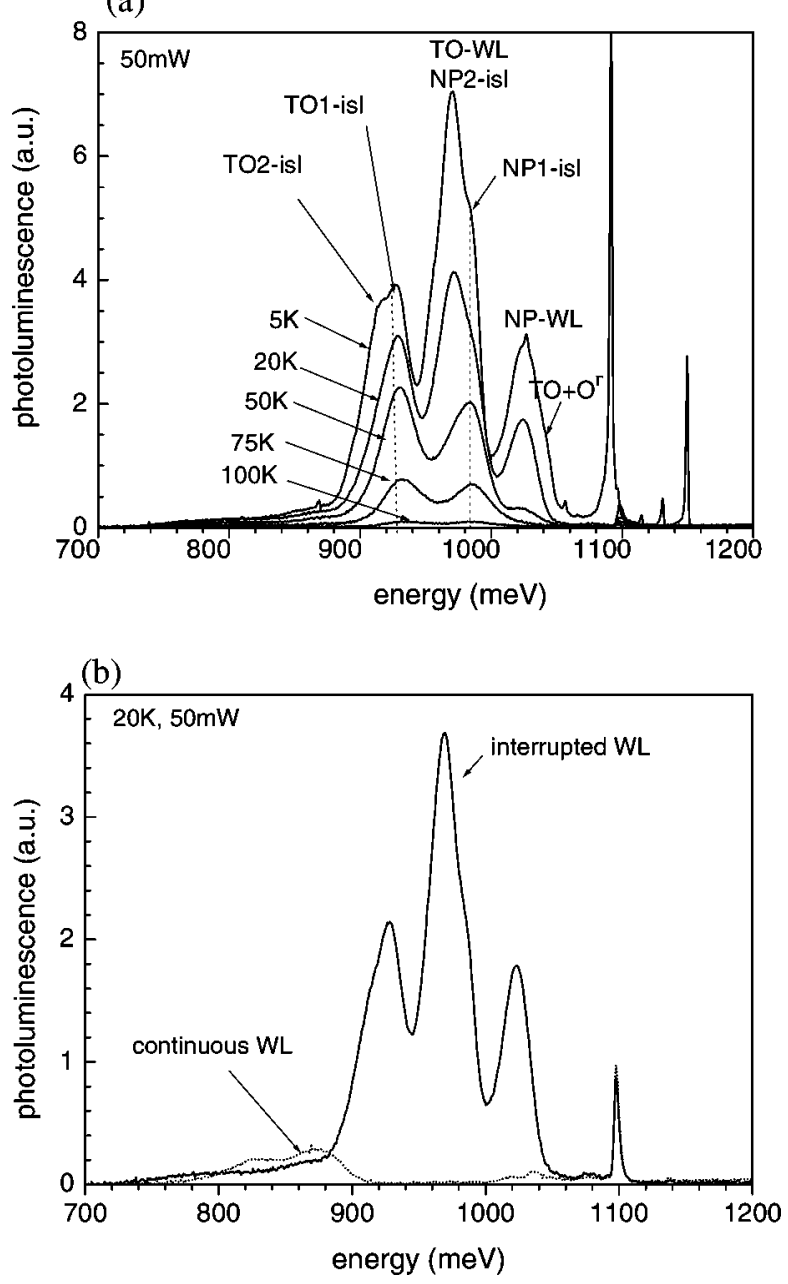

FIG. 3. Photoluminescence of capped islands (a) for a sample with islands only in voids similar to sample of Fig. 1(a), (b) comparison with a second sample with islands on the whole surface (continuous wetting layer).

both, pyramids and domes, we have taken interdiffusion into account (due to the high deposition temperature of $700^{\circ} \mathrm{C}$ ) leading to a Si content of $\sim 50 \%$ and $\sim 65 \%$, respectively, as was shown by De Seta et al. ${ }^{11}$

The model has to be regarded critically. Values for $h_{\mathrm{SK}}$, wetting layer thickness $\left(d_{\mathrm{WL}}\right)$ after the SK transition has set in and $\mathrm{Si}$ content in islands nucleated on small areas are not yet known. Even so, the model can be a guide for the design of island deposition on small patterns. The lateral diffusion is not supposed to influence this phenomenon under the present conditions because it is much longer than the pad size studied here at the same deposition temperature. ${ }^{8}$ After attaining the $h_{\mathrm{SK}}$ one or more islands are formed if the amount of Ge in a void corresponds to one or more islands. If the amount is smaller than $2 \times V_{\text {isl }}$ than only one island is formed.

On samples with voids or windows the Ge wetting layer (WL) is not continuous. We expect the photoluminescence to change as compared to large areas. ${ }^{12}$ Figure 3 displays the spectral distribution of PL of a wafer with interrupted WL in comparison with a wafer prepared in the same run but with continuous WL. The Ge coverage was $0.68 \mathrm{~nm}$ and the $\mathrm{Si}$ cap layer thickness was $30 \mathrm{~nm}$. We attribute the high PL intensity to the interruption of the wetting layer, i.e., excitons can diffuse laterally only within the void, in contrast to the sample with continuous WL where excitons are free to migrate hundreds of microns along WL. ${ }^{8}$ The long migration length of excitons along the WL in large area samples is one of the reasons for the low radiative recombination from islands. In Fig. 3(a) the PL is a superposition of three pairs of peaks all lying at higher energy than the emission from the continuous area part $3 \mathrm{~b}$ and usually observed on large areas under similar conditions. ${ }^{5}$ To understand the high intensity and the peak positions we have analyzed the island distribution of an uncapped sample. We observe that the density of islands is several times lower than on extended areas with the oxide completely removed. This is in this particular case so because the island density is given by the void density $\left(0.4 \mu \mathrm{m}^{-2}\right)$. Therefore, in spite of the much smaller number of islands contributing to the emission from the sample with voids, the intensity is much higher than from the sample with extended WL. Moreover, the island height lies in the range $h=4-8 \mathrm{~nm}$, while most of the islands of samples without oxide have islands with $h=6-25 \mathrm{~nm}$. Thus, the small height of the islands in voids must be responsible for the high energetic position of the PL.

In conclusion, we have studied the dependence of number of islands deposited by selective epitaxial growth on small and finite areas. The number increases by one only after a certain coverage and above a certain area size. The arrangement of islands near the periphery of the voids is supposedly driven by the elastic distortion of $\mathrm{Si}$ near the periphery of the oxide window.

${ }^{1}$ E. S. Kim, N. Usami, and Y. Shiraki, Appl. Phys. Lett. 72, 1617 (1998).

${ }^{2}$ Y. Nitta, M. Shibata, K. Fujita, and M. Ischikawa, Surf. Sci. 462, L587 (2000).

${ }^{3}$ M. Liehr, J. E. Lewis, and G. W. Rubloff, J. Vac. Sci. Technol. A 5, 1559 (1987).

${ }^{4}$ T. Couteau, M. McBride, and D. Riley, Semicond. Int., 95 (1998).

${ }^{5}$ L. Vescan, M. Goryll, T. Stoica, P. Gartner, K. Grimm, O. Chretien, E. Mateeva, C. Dieker, and B. Holländer, Appl. Phys. A: Mater. Sci. Process. 71, 423 (2000).

${ }^{6}$ L. H. Nguyen, V. Le Thanh, D. Debarre, V. Yam, and D. Bouchier (unpublished).

${ }^{7}$ T. I. Kamins and R. S. Williams, Appl. Phys. Lett. 71, 1201 (1997).

${ }^{8}$ L. Vescan, J. Phys.: Condens. Matter 14, 8235 (2002).

${ }^{9}$ S. M. Hu, J. Appl. Phys. 70, R53 (1991).

${ }^{10}$ M. Krishnamurthy, B.-K. Yang, and J. D. Weil, Appl. Phys. Lett. 70, 49 (1997).

${ }^{11}$ M. De Seta, G. Capellini, F. Evangelisti, and C. Spinella, J. Appl. Phys. 92, 614 (2002).

${ }^{12}$ L. Vescan and T. Stoica, J. Appl. Phys. 91, 10119 (2002). 